\title{
The Chromatin Remodeler Mi2/CHD4 is Required in Skeletal Muscle Stem Cells for Normal Muscle Development and Regeneration
}

\author{
Assia Derfoul \\ National Institutes of Health \\ lago Pinal-Fernandez \\ National Institutes of Health \\ Wilson Huang \\ National Institutes of Health \\ Cassie Parks \\ National Institutes of Health \\ Katherine Pak \\ National Institutes of Health \\ Victoria Hoffmann \\ National Institutes of Health \\ Andrew Mammen ( $\nabla$ andrew.mammen@nih.gov ) \\ National Institutes of Health \\ Katia Georgopoulos \\ Harvard University
}

\section{Research Article}

Keywords: CHD4, DNA, NuRD, Embryonic

Posted Date: December 2nd, 2020

DOI: https://doi.org/10.21203/rs.3.rs-112107/v1

License: (1) (1) This work is licensed under a Creative Commons Attribution 4.0 International License.

Read Full License 


\title{
The chromatin remodeler Mi2/CHD4 is required in skeletal muscle stem cells for normal muscle development and regeneration
}

\author{
Assia Derfoul ${ }^{1}$, Iago Pinal-Fernandez ${ }^{1}$, Wilson Huang ${ }^{1}$, Cassie Parks ${ }^{1}$, Katherine Pak ${ }^{1}$, Victoria \\ J. Hoffmann ${ }^{2}$, Katia Georgopoulos ${ }^{3}$, and Andrew L. Mammen ${ }^{1}$
}

\begin{abstract}
${ }^{1}$ Muscle Disease Unit, Laboratory of Muscle Stem Cells and Gene Regulation, National Institute of Arthritis, Musculoskeletal \& Skin Diseases, National Institutes of Health, Bethesda, MD 20892, USA.

${ }^{2}$ Office of Research Services, Division of Veterinary Resources, National Institutes of Health, Bethesda, MD 20892, USA.

${ }^{3}$ Cutaneous Biology Research Center, Massachusetts General Hospital, Harvard Medical School, Charlestown, MA 02129, USA.
\end{abstract}

Address correspondence to: Andrew L. Mammen, M.D., Ph.D., Muscle Disease Unit, Laboratory of Muscle Stem Cells and Gene Expression, National Institute of Arthritis and Musculoskeletal and Skin Diseases, National Institutes of Health, 50 South Drive, Room 1141, Building 50, MSC 8024, Bethesda, MD 20892. E-mail: andrew.mammen@nih.gov

Phone: 301-451-1199. Fax: 301-594-0305. 


\begin{abstract}
The chromodomain helicase and DNA binding 4 (CHD4) protein is upregulated in regenerating myofibers. To define the role of CHD4 in muscle differentiation and regeneration, we generated mice with CHD4 ablated in muscle satellite cells (SCs). Embryonic day 18.5 CHD4 KO mice are non-viable, with atrophic intercostal and back muscles and altered expression of muscle contraction genes. Tamoxifen-inducible conditional CHD4 KO in adult mouse SCs diminished myoblast proliferation, induced premature differentiation, and altered expression of muscle contraction genes at the myotube stage. Following cardiotoxin-induced muscle injury, $\mathrm{CHD} 4 \mathrm{KO}$ regenerating myofibers had reduced cross-sectional area. ChIP-Seq analysis revealed that CHD4 binds actin $\alpha 1$, Wnt and $\beta$-catenin genes, which are known to play roles in the regulation of myogenesis. Together, our results suggest an important role for CHD4 in the control of embryonic myogenesis, SC differentiation, and the control of muscle fiber size in adult skeletal muscle during regeneration.
\end{abstract}




\section{Introduction}

Chromatin helicase DNA binding 4 (CHD4) encodes a chromatin remodeling enzyme belonging to the SNF2 superfamily of ATPases. CHD4 is expressed in developing and adult tissues including the skin, kidney, thymus, liver, areas of the brain, hair follicles, and mucosal epithelia. CHD4 is a core member of the nucleosome remodeling and histone deacetylation (NuRD) complex, which couples CHD4 helicase activity to the activity of histone deacetylases HDAC1 and HDAC2. Because the NuRD complex contains several proteins associated with transcriptional repression (including $\mathrm{HDAC} 1 / 2$, histone chaperones retinoblastoma binding protein $\mathrm{RBp} 46$ and RBp48, DNA-binding proteins MTA1 and MTA2, and methyl CPG binding domain proteins MBD2 and MBD3) it was initially thought that CHD4 establishes a repressive chromatin structure. However, numerous studies have shown that CHD4 can be associated with other regulatory functions ${ }^{1}$, including transcriptional activation ${ }^{2-5}$.

In mice, knockout (KO) of CHD4 is lethal due to the inability of embryos to successfully form the trophectoderm, which is required for blastocyst implantation ${ }^{6}$. However, tissue-specific CHD4 KO mice have allowed for analyses showing that CHD4 plays an important role in the differentiation of epidermal and neuronal progenitor cells as well as in T and B-cell development $2,7-10$.

We have previously shown that CHD4 levels are increased in skeletal muscle cells during muscle regeneration ${ }^{11,12}$. Furthermore, we showed that CHD4 silencing in the embryonic mouse muscle $\mathrm{C} 2 \mathrm{C} 12$ cell line leads to accelerated myoblast differentiation ${ }^{11}$. In vivo, a recent study showed that CHD4 deletion in muscle stem cells results in defective muscle regeneration due to loss of repression of the necroptosis effector Ripk3 ${ }^{13}$. Interestingly, deletion of CHD4 in terminally differentiated skeletal muscle fibers was shown to result in aberrant upregulation of cardiac muscle myogenic differentiation genes and a myopathy ${ }^{14}$, suggesting that CHD4 is required for the maintenance of adult skeletal muscle fiber identity and homeostasis. However, the role of CHD4 in skeletal muscle during development has not been explored. Therefore, in this study, we have examined the effects of CHD4 deletion in skeletal muscle stem cells, also known as satellite cells (SCs), during myogenesis. These studies demonstrate that deletion of CHD4 in SCs during development alters the expression of muscle development genes and results in the defective formation of back and intercostal muscles. Furthermore, we show that deletion of CHD4 from SCs 
in adult mice leads to impaired muscle cell differentiation in vitro and defective muscle regeneration following cardiotoxin muscle injury in vivo. 


\section{RESULTS}

\section{Conditional deletion of CHD4 in Pax-7-derived muscle precursor cells during embryogenesis}

is lethal

To study its functional role during skeletal muscle development, we conditionally ablated CHD4 in SCs, the stem cells which give rise to skeletal muscle. Specifically, we crossed knock-in mice expressing Cre recombinase from the Pax7 locus (Pax7-Cre) ${ }^{15}$ with animals bearing floxed CHD4 alleles ${ }^{2}$ to generate Pax7-Cre ${ }^{+/-}$; CHD4 $4^{\mathrm{fl} / f 1}$ mice (abbreviated CHD4 muscle KO, $\mathrm{CHD} 4 \mathrm{mKO}$ ). In crosses predicted to generate $25 \%$ of $\mathrm{CHD} 4 \mathrm{mKO}$ mice, genotyping at weaning revealed no surviving $\mathrm{CHD} 4 \mathrm{mKO}$ offspring, suggesting that CHD4 deletion is lethal during embryogenesis or shortly after birth. Genotyping of day 18.5 embryos (E.18.5) by PCR of the WT and the deleted CHD4 alleles (Fig. 1A) showed that CHD4mKO embryos were present in the predicted Mendelian ratios (Fig. 1B), suggesting that these mice survive embryogenesis but die soon after birth. Indeed, we observed that, unlike WT or heterozygous CHD4 KO mice, CHD4mKO day 18.5 embryos had apparent respiratory distress and died shortly after collection (Fig. 1C).

\section{CHD4 knockout results in the underdevelopment of intercostal and thoracic musculature}

Immunofluorescence analysis of the hindlimb muscles derived from E.18.5 embryos showed that most muscle nuclei from CHD4mKO embryos lack CHD4 expression (Fig 1D). Whole-mount histological analysis of E.18.5 CHD4mKO embryos revealed no gross morphological changes in the hindlimb muscles (Sup. Fig1A). However, the neck, back (midthorax and cranial thorax), and intercostal muscles were consistently underdeveloped when compared to those of WT embryos (fig1E and Sup Fig1B). For example, the width of the intercostal (IC) and thoracic muscles in the CHD4mKO embryos was reduced relative to WT (Fig. 1E, Sup. Fig1A) and muscle fibers were less densely packed (Fig. 1E). In contrast, no changes were observed in the diaphragm oh the CHD4mKO embryos (data not shown).

\section{Transcriptomic changes in skeletal muscle caused by knockout of CHD4}

To examine changes in the transcriptome produced by the ablation of CHD4 during embryonic myogenesis in different muscle groups, we performed RNASeq analysis of the 
intercostal, back, and hindlimb muscles of CHD4mKO and WT embryos. The number of unique and shared differentially expressed genes for each of these muscles is represented in a Venn diagram in Sup Fig 1C. Consistent with the disrupted development of intercostal muscle in CHD4mKO embryos, GO analysis showed that the most downregulated biological process pathway was that of muscle contraction $\left(P=9.65 \times 10^{-22}\right)$ (Fig 1F). Downregulated genes in this pathway include two notable transcriptional factors, myogenic factor 6 (Myfo) and four and a half limb domains 1 (FHL1), both of which are known to play important roles in muscle development. In addition, a number of structural proteins such as actin $\alpha 1$ (ACTA1), myosin heavy chain 8 $(M y h 8)$ and dystrophin $(D M D)$, had significantly reduced expression in the intercostal muscles of CHD4mKO embryos (Sup. Table 3). GO analysis of upregulated genes in the intercostal muscle of CHD4mKO embryos revealed upregulation of the lipid metabolic process pathway ( $P=4.1 \times 10^{-}$ $\left.{ }^{4}\right)$ (Fig. 1F) which included upregulation of phospholipase BD1 (PLBDI) and apolipoprotein E $(A P O E)$ as well as a number of enzymes associated with acetyl-CoA metabolism (ACAA1B, $A C A T 3$ and HACL1) (Sup. Table 3). These observations suggest that CHD4 KO intercostal muscle has altered phospholipid catabolism and increased oxidative phosphorylation. Gene set enrichment analysis (GSEA) analysis of the differentially expressed genes in the intercostal muscle confirmed that muscle contraction genes were altered in the CHD4mKO in this muscle group (Fig 1F, lower panel).

In the back muscles, $\mathrm{GO}$ analysis revealed that not only muscle structural protein genes but also genes encoding mitochondrial proteins (Fig 1G) were significantly downregulated in CHD4mKO embryos, suggesting an alteration of both muscle contraction and energy homeostasis in this muscle group. Several genes, including the muscle-specific genes Myh8, MYO5C, Myoz1, $M y o z 3$ and MYBPC2, were significantly downregulated in the back muscles of $\mathrm{KO}$ animals (Sup. Table 3) even though GSEA analysis for the muscle contraction pathway in the back muscles did not reach significance (Fig 1G, lower panel). Together, these results suggest that CHD4 is essential for muscle structure and function and that its deletion leads to the perturbation of muscle contraction, energy homeostasis, and myopathy of the back and intercostal muscles. We hypothesize that these skeletal muscle defects are responsible for the respiratory distress which leads to the death of CHD4mKO animals.

Although histological analysis of E.18.5 CHD4mKO mice showed no changes in the hindlimb muscles (Sup Fig1A), RNASeq analysis identified 164 differentially expressed genes in 
hindlimb muscles from the CHD4mKO mice; 120 of these were differentially expressed in the hindlimb muscles, but not the intercostal or back muscles (Sup Fig.1C). The top GO enriched pathways for the upregulated genes consisted of calmodulin-binding and glycoprotein genes whereas extracellular matrix and mitochondrion were the most enriched pathways for downregulated genes (Sup Fig 1D). Notably, Wnt family members Wnt4 and Wnt16, known to play a key role in myogenesis during development and regeneration ${ }^{16}$, were suppressed while growth differentiation factor 11 (GDF11), known for its role as a negative regulator of muscle development, was significantly upregulated (Sup.Table 3). Thus, despite the absence of phenotypic changes, upstream regulators of structural and metabolic muscle functions were both altered in hindlimb muscles of the CHD4mKO mice.

\section{Conditional deletion of CHD4 in adult satellite cells results in defective differentiation of myoblasts into myotubes}

Since postnatal skeletal muscle growth and regeneration are driven by SCs, we examined CHD4 expression in WT SCs, MBs, and MTs. SCs were isolated by FACS from WT animals as previously described ${ }^{17}$ and were either fixed immediately as quiescent SCs (day 0) or cultured for two days in GM or DM to allow differentiation into MBs or MTs. Immunofluorescence analysis showed that CHD4 is expressed in quiescent SCs and co-localizes with the nuclear SC marker Pax-7 (Fig 2A). As cells differentiate into MBs, CHD4 is co-expressed with myogenin (Myog). However, when cells proceed to form myotubes expressing myosin heavy chain (Myhc), CHD4 expression is downregulated compared to MBs (Fig 2B).

We next used a tamoxifen-inducible system to generate a conditional muscle CHD4 KO in adult mice by crossing the knock-in mice expressing Cre-ER ${ }^{\mathrm{T} 2}$ recombinase from the Pax7 locus $\left(\right.$ Pax7-Cre ER ${ }^{\mathrm{T} 2}$ ) with animals bearing floxed CHD4 alleles to generate the Pax7-Cre ER ${ }^{\mathrm{T} 2+/}$, $\mathrm{CHD}^{\mathrm{fl} / \mathrm{fl}}$ mice (Fig. 2C, Left panel). Upon tamoxifen administration, expression of CRE recombinase is activated in Pax-7 expressing-SCs leading to the ablation of CHD4 in the muscle of Pax-7 expressing SCs and the generation of Tam-CRE muscle CHD4 KO (abbreviated TamCRE CHD4mKO). At 15- and 21-days post-tamoxifen injection, SCs from both WT and KO mice were isolated by FACS (Fig. 2C, right panel). The total SC numbers were equivalent between WT and KO mice (Fig. 2D). However, RT-qPCR and immunofluorescence studies revealed that CHD4 
expression was reduced by about 50\% in the KO compared to WT mice (Fig. 2E \& 2F), indicating that CHD4 KO in adult SCs was substantial but not complete.

To explore changes in the Tam-CRE CHD4mKO SCs at the transcriptome level, we performed RNASeq analysis on both WT and CHD4 KO cells. Under MB growth conditions for 48h, GO analysis of the upregulated genes in KO cells showed that the top enriched pathways included cell adhesion $\left(P=2.49 \times 10^{-11}\right)$, TGF- $\beta$ signaling pathway $\left(P=5.08 \times 10^{-6}\right)$, and skeletal system development $\left(P=2.31 \times 10^{-5}\right)$ while downregulated genes in $\mathrm{KO}$ cells enriched for cell cycle and cell division pathways (Fig. 2G). Notably, expression of actinin $2 / 3$ and titin, which are expressed at high levels in MTs, were significantly upregulated in Tam-CRE CHD4mKO MBs (Sup. Table 4). In contrast, GO analysis of the downregulated genes in CHD4 KO MBs enriched for cell cycle and $\left(\mathrm{P}=4.92 \times 10^{-74}\right)$ and cell division $\left(\mathrm{P}=8.53 \times 10^{-56}\right)$ (Fig. 2G). For example, genes expressed at high levels in proliferating cells, including cyclin E (CcnE), cyclin B1 (Ccnbl) and cyclin dependent kinase $1(C d k l)$, were reduced in the KO cells (Sup. Table 4). These findings suggest that CHD4 KO causes reduced SC proliferation and an acceleration of SC differentiation.

In SCs induced to differentiate into MTs, GO analysis revealed an upregulation of genes associated with the extracellular matrix pathway $\left(P=3.1 \times 10^{-12}\right)$ ( Fig. $\left.2 \mathrm{H}\right)$, including a large number of collagens (Colala1, Col2a1, Col6a1, Colsa2, Coll1al and Col12al), matrix metalloproteases ( $M m p 9$, Mmp14 and Mmp17), fibrillin1 (FBN1), and transforming growth factor beta1 and 2 (TGF $\beta 1$ and $T G F \beta 2$ ) (Sup. Table 4). Downregulated genes in CHD4 KO MTs included those associated with mitochondrion $\left(P=1.28 \times 10^{-09}\right), \mathrm{Z}$ disc $\left(P=1.55 \times 10^{-5}\right)$ and myofibril $\left(P=5.95 \times 10^{-5}\right)$ GO pathways (Fig $\left.2 \mathrm{H}\right)$. For example, expression levels of pyruvate dehydrogenase A1 (PDHA1) and acetyl-CoA acyltransferase (ACAA2) (mitochondrial), which are associated with muscle energy metabolism, were downregulated (Sup. Table 4). Similarly, genes involved in muscle contraction, such as myosin heavy chain 6 and 7 (Myh6 and Myh 7) were also significantly reduced (Sup. Table 4). Taken together, these data suggest that Tam-CRE CHD4 mKO MBs have diminished proliferative capacity and differentiate faster than WT MBs, but lack of CHD4 results in incomplete differentiation program at the MT stage.

\section{CHD4 binds to muscle, Wnt/ $\beta$-catenin and metabolic genes during myogenesis}

To identify genes that are directly regulated by CHD4, we performed chromatin immunoprecipitation followed by high throughput sequencing (ChIP-Seq) using anti-CHD4 antibodies and 
chromatin from the murine $\mathrm{C} 2 \mathrm{C} 12$ myogenic cell line at the MB and MT stages. In $\mathrm{C} 2 \mathrm{C} 12 \mathrm{MBs}$, CHD4 bound mostly to introns (16.2\%) and intergenic regions (74.4\%) and less so to promotor regions (2.6\%). In $\mathrm{C} 2 \mathrm{C} 12 \mathrm{MTs}, \mathrm{CHD} 4$ bound promoters much more frequently $(15.2 \%)$ than in MBs (Fig. 3A). Interestingly, CHIP-seq revealed CHD4 peaks covering the entire coding region of the skeletal muscle actin $\alpha 1$ (Actal) gene in MBs and in MTs (Fig. 3B). Since Actal is downregulated in muscles from CHD4 KO mice, this finding would suggest that CHD4 may directly promote Actal expression. Although we observed similar downregulation of Actal expression in CHD4 KO SCs MB and MT as well as C2C12 CHD4 siRNA knock down (KD) by RNASeq (data not shown), these differences did not reach significance. This observation could result from the incomplete $\mathrm{KO}$ (Fig 2E) and KD (data not shown) of CHD4 in these experimental settings. Similarly, CHIP-Seq showed CHD4 peaks at the $\beta$-catenin promoter-TSS and Wnt11 intergenic regions in MTs (Fig. 3C). As activation of the Wnt signaling pathway antagonizes Notch signaling and thereby leads to increased terminal differentiation ${ }^{18,19}, \mathrm{KO}$ of CHD4 would also be expected to accelerate differentiation if CHD4 normally represses expression of Wnt. Finally, we observed a similar pattern of CHD4 interaction on CHIP-Seq for cytochrome P450, family 27, subfamily a (Cyp27a) and acetyl-Coenzyme A carboxylase beta (Acacb) which are both involved in cholesterol metabolism and fatty acid oxidation, respectively (Fig. 3D). Taken together, the ChIP-Seq results are consistent with the CHD4 functioning as a direct regulator of gene expression during muscle cell differentiation.

As previously described in other cells where CHD4 represses transcription of the $\gamma$-globin

gene ${ }^{20-23}$ CHD4 also bound to genes encoding a number of erythroid-specific factors including erythroid differentiation regulator 1, hemoglobin alpha, adult chain 2 and hemoglobin, and beta adult s chain (EDRl-, Hba-a2 and Hbb-bs, hematopoietic) (Fig. 3E and data not shown) in both MBs and MTs. In addition, CHD4 bound POU domain, class 4, transcription factor 1 (Pou4fl, neuronal), as well as myosin 7a (MYO7a, cardiac) (data not shown) genes.

\section{Conditional deletion of CHD4 in adult SCs disrupts muscle regeneration}

To examine the effect of CHD4 KO on the function of SCs in vivo, we induced muscle regeneration by injecting the left TA muscle of both WT and KO Tam-CRE CHD4 animals with cardiotoxin (CTX) following tamoxifen administration (Fig. 4A). CHD4 immunostaining of 
injured TA muscle cross sections showed that $\sim 50 \%$ of the Tam-CRE CHD4mKO regenerated fibers did not express CHD4 (Fig 4B and 4C), consistent with the incomplete penetrance of the CHD4 KO observed in SCs in vitro (Fig 2). Ten days following muscle injury (CTX D10), we observed an overexpression of embryonic Myh (Myhemb) in the regenerating muscles of Tam-CRE CHD4mKO mice compared to the WT mice (Fig. 4D), suggesting altered muscle regeneration in the absence of CHD4 expression. A quantitative analysis of regenerating fiber size revealed an increased number of small fibers at all days following injury (days 7, 10, 14 and 28) in the TamCRE CHD4mKO compared to the WT mice (Fig. 4E and 4F and data not shown). Notably, TamCRE CHD4mKO muscle had significantly higher numbers of fibers with 6-10 $\mu \mathrm{M}$ diameter and smaller numbers of fibers with 16-20 $\mu \mathrm{M}$ diameter even 28 days following CTX injury compared to injured WT muscle (Figure 4G). Furthermore, the total myofiber number in the Tam-CRE CHD4mKO mice was more than two-fold higher than in the WT mice (Figure 4H).

Transcriptomic analysis of regenerating TA muscle at all days surveyed following injury (day 7, 10, 14 and 28), showed that a number of genes, including several muscle-specific and metabolic genes, were differentially expressed in the Tam-CRE CHD4mKO compared to WT muscle (Sup. Table 5). Notably, the gene for embryonic myosin heavy chain (Myh3) was upregulated at day 14 post-CTX. In contrast, nexilin F-actin binding protein (Nexn), which has an essential role in the maintenance of the $\mathrm{Z}$ line and sarcomere integrity, was downregulated at day 7 post-CTX (Sup. Table 5). In addition, the energy and metabolic genes, estrogen related receptor alpha $(E S R R \alpha)$ and glucokinase $(G c K)$ were upregulated in the Tam-CRE CHD4mKO muscle at day 7 and 14 post-CTX, respectively (Sup. Table 4), suggesting alteration of both glucose and fat metabolism in the injured CHD4 KO muscle. 


\section{Discussion}

CHD4/NuRD is known to play a role in the differentiation of stem cells as they give rise to a number of different tissues ${ }^{2,7-9}$. However, the function of CHD4 in skeletal muscle stem cells during embryonic development has not been explored. In this study, we show that conditional deletion of CHD4 in SCs during development results in alteration of the skeletal muscle differentiation transcriptional program along with an impaired development of intercostal and back muscles which likely leads to early postnatal death due to respiratory failure. Although epaxial (back) and hypaxial (intercostal) muscles were affected in the CHD4mKO mice, no differences were observed in the hypaxial muscles of the tongue, diaphragm or limb, which originate from the lateral plate mesoderm. It is well established that during development, the back (epaxial), abdominal and intercostal (hypaxial) muscles derive from muscle precursor cells (MPCs) of epithelial origin. In contrast, the tongue, diaphragm and limb (hypaxial) muscles derive from migratory $\mathrm{MPCs}^{24}$. Our results would suggest that CHD4 may not be required for development of muscles deriving from embryonic migratory MPCs.

In both the intercostal and back muscles of E18.5 CHD4mKO mice, we observed a downregulation of genes encoding skeletal muscle proteins that correlated with the observed myopathy in these muscles. In addition, the observed downregulation of mitochondrial genes in the back and upregulation of lipid metabolism in the intercostal muscle suggests alteration of energy homeostasis in the CHD4mKO muscle. Interestingly, GDF11 upregulation and Wnt 4/16 downregulation was a shared consequence of CHD4 $\mathrm{KO}$ in all tested muscle groups, including the intercostal, back, and hindlimb muscles. A member of the transforming growth factor beta (TGF$\beta$ ) family, GDF11 has been shown to inhibit myoblast proliferation and differentiation, delay muscle regeneration, and promote muscle atrophy ${ }^{25-27}$. In contrast, the $W n t$ gene superfamily encodes glycoproteins which bind to the Frizzled $(F z d)$ transmembrane receptors on target cells and thereby regulate key differentiation processes during embryonic myogenesis ${ }^{28-31}$. The combination of GDF11 upregulation along with downregulation of $W n t$ signaling suggests that the intercostal and back muscle abnormalities in CHD4 KO embryos could be mediated, in part, by perturbing these pathways. A recent study showed that deletion of CHD4 in adult muscle stem cells leads to increased expression of Ripk3, which mediates satellite cells necroptosis ${ }^{13}$. Our RNA-Seq data did not reveal upregulation of Ripk3 in E18.5 CHD4mKO muscles suggesting that 
CHD4-induced Ripk3 suppression is not required for muscle stem cells proliferation and differentiation during muscle development.

Similar to our findings, cardiomyopathy has recently been reported in mice with deletion of CHD4 in cardiac muscle progenitor cells ${ }^{14,25,32}$. Interestingly, CHD4 deletion in the heart muscle progenitor cells during development resulted in the aberrant upregulation of genes typical of skeletal muscle ${ }^{14}$. However, we did not find aberrant expression of cardiac genes in our skeletal muscle specific CHD4mKO mice.

Myogenic differentiation is a highly orchestrated process, with MBs undergoing cell cycle withdrawal before expressing contractile proteins and fusing to form MTs ${ }^{33}$. CHD4's role in promoting cell proliferation has been previously demonstrated in other cell types ${ }^{34,35}$. In the current study, we found that CHD4 KO in SCs resulted in down-regulation of cell cycle genes and increased activation of the TGF- $\beta$ signaling pathway, which is known to inhibit SC and myoblast proliferation ${ }^{36}$. We hypothesize that $\mathrm{CHD} 4 \mathrm{KO}$ causes reduced MB proliferation and premature MB differentiation with increased levels of muscle proteins, myofibril and sarcomere components. The inability of the CHD4 KO myoblasts to activate genes essential for myotube formation when transferred to differentiation medium suggests that CHD4 is required for this process in vitro.

In vivo, the total SC numbers were equivalent between WT and Tam-CRE CHD4 mKO mice. This is in disagreement with a recent study that showed a reduced number of SCs in the CHD4 $\mathrm{KO}^{13}$, which could be explained by the partial $\mathrm{KO}$ of the CHD4 protein we obseve in our study following tamoxifen administration. Histologically, no differences were noted between the TA muscle fibers from Tam-CRE CHD4 mKO mice compared to WT mice (data not shown). However, following muscle regeneration induced by muscle injury, there was an increased number of total myofibers and a higher proportion of smaller myofibers in Tam-CRE CHD4mKO at all time points tested. We propose that this may have resulted from an accelerated differentiation of CHD4 KO SCs and early fusion of regenerating fibers leading to an increased number of smaller fibers. Of note, an association of early differentiation with smaller fiber size during muscle regeneration has been shown to occur in the context of increased canonical $\mathrm{Wnt} / \beta$-catenin-induced follistatin (Fstll) levels ${ }^{37}$. In this regard, it is of interest that our ChIP-Seq data showed that CHD4 was enriched at the $\beta$-catenin promoter-TSS and Wnt11 intergenic regions in MTs. In addition, deletion of CHD4 resulted in the upregulation of Wnt family members and follistatin (Fstl1) in CHD4 KO SCs differentiated in vitro (Sup. Table 5). Wnt signaling has been shown to be 
important for myogenesis in adult SCs and failure to silence it interferes with muscle regeneration 19. Together, our results suggest an important role for CHD4 in the control of embryonic myogenesis and in the regulation of SC differentiation as well as the control of muscle fiber size in the adult skeletal muscle during regeneration. 


\section{Methods}

\section{Animals}

All animals were housed in a pathogen-free facility and all experiments were performed according to the National Institutes of Health (NIH) Animal Care and Use regulations. The Pax7-Cre ${ }^{15}$ and Pax7-CRE ${ }^{\text {ERT2 }}$ mice were obtained from Jackson Labs. Mice with floxed CHD4 alleles have been previously described ${ }^{38}$ and were obtained from Dr. Katia Georgopoulos. All mouse experiments were performed in accordance with protocol A017-11-07 authorized by the National Institute of Arthritis and Musculoskeletal and Skin Diseases/National Institutes of Health Animal Care and Use Committee. Genomic DNA was prepared from tail muscle tissue and genotyping was performed by polymerase chain reaction (PCR). Specific oligonucleotide sequences used as primers and PCR conditions are listed in supplementary (Sup) Table 1).

\section{Muscle injury}

Eight to twelve-week old WT and CHD4mKO mice were injected with tamoxifen for four consecutive days, allowed to rest for three days, then anesthetized with isoflurane such that there was no response to tactile stimuli. The left lower extremity was shaved, and the tibialis anterior (TA) muscle was injected with $100 \mu 1$ of $10 \mathrm{mM}$ cardiotoxin (CTX; Calbiochem). The uninjected right TA muscle served as a control. Mice were euthanized at 7, 10, 14- and 28 days post-injury ( $\mathrm{n}=2-4$ mice/genotype/time point) and both TA muscles were carefully excised and mounted on tragacanth (Sigma) loaded cork disks; frozen sections were used for RNA, histology and immunohistochemistry analyses.

\section{Histology and immunohistochemistry}

E18.5 whole embryos were fixed in $10 \%$ formalin at room temperature, embedded in paraffin, sectioned, and stained with hematoxylin and eosin $(H \& E)$. Stained sections were examined by the Pathology Service of the Division of Veterinary Resources at NIH. Hind limb, intercostal and spinal muscles were dissected from an additional E18.5 embryo and either stored in RNA Later or embedded in OCT compound for RNA preparation and immunostaining, respectively. Control and injured TA muscles were dissected from adult WT and CHD4 KO mice and mounted on tragacanth (sigma) loaded on cork discs. E18.5 hind limb and adult control and injured TA muscles were cryo-sectioned at $10 \mu \mathrm{M}$ and processed for H\&E staining as well as 
immunohistochemistry. For immunofluorescence, sections were fixed in freshly prepared $4 \%$ paraformaldehyde followed by an antigen retrieval step performed in 1X citrate buffer (Invitrogen) at high pressure, in a pressure cooker for 10 minutes. Primary and secondary antibodies used, and their dilution conditions are listed in Sup. Table 2. Immunofluorescence images were captured using Leica DM6000 Zeiss or Axiovert S100 fluorescence microscopes. For fiber size analyses, six images of a laminin stained section were captured blindly for each TA section and crosssectional fiber area was determined from a randomly chosen image using ImageJ software. Data collected from D7, 10, 14 and 28 injured TA muscles ( $\mathrm{n}=2-4$ mice/genotype/time point) was analyzed using a script generated in Python software.

\section{Satellite cell preparation and FACS}

Satellite cells were prepared and FACS-sorted as previously described ${ }^{17}$. Skeletal muscles from 8-12 weeks old mice were dissected from both hind limbs and torn with forceps then digested with collagenase type 2 (Worthington, $2.5 \mathrm{U} / \mathrm{ml}$ ) for $30 \mathrm{~min}$ at $37^{\circ} \mathrm{C}$. Following washing with PBS, a second digestion was performed with collagenase B (Roche Biochemicals $2.5 \mathrm{U} / \mathrm{ml}$ ) and dispase (Roche Biochemicals $2.4 \mathrm{U} / \mathrm{ml}$ ) for 1 hour at $37^{\circ} \mathrm{C}$. Digestion reactions were stopped with $2 \mathrm{mM}$ EDTA and cell preparation was diluted with PBS, passed through an $18 \mathrm{G}$ syringe ten times then filtered through a $40 \mu \mathrm{m}$ cell strainer. Cells were collected by centrifugation at $400 \mathrm{~g}$ for $5 \mathrm{~min}$ then counted. For fluorescence-activated cell sorting (FACS), cell preparation was re-suspended in PBS supplemented with $15 \%$ heat-inactivated FBS at $1 \times 10^{7}$ cells $/ \mathrm{ml}$ and incubated for $30 \mathrm{~min}$ at $4^{\circ} \mathrm{C}$ with the following primary antibodies: anti-Cd11b, anti-CD31, anti-CD45 and anti-Sca-1 (BD Biosciences) conjugated to fluorescein isothiocyanate (FITC) in addition to anti- $\alpha 7$-integrin conjugated to phycoerythrin (PE)(MBL). Complete antibody information is described in supplemental Tabe 2. To select for viability and exclude fiber debris, cells were co-stained with $1 \mathrm{mg} / \mathrm{ml}$ propidium iodide (PI) and $2.5 \mathrm{mg} / \mathrm{ml}$ Hoechst (Molecular Probes) and cells were resuspended at $1 \times 10^{7}$ cells $/ \mathrm{ml}$ immediately before sorting. For all antibodies, we performed fluorescence minus one controls as well as single stain controls. Cell sorts were performed on an Influx or a FACSAria Fusion (Becton and Dickenson) equipped with three lasers using a $100 \mu \mathrm{m}$ nozzle. Data was collected with FacsDIVA software and bioexponential analysis was performed using FlowJo 9.1 (Treestar) software. 


\section{Cell culture}

C2C12 cells obtained from ATCC were maintained in DMEM supplemented with 10\% FBS and 2\% penicillin and streptomycin (Invitrogen) growth medium. For differentiation experiments $\mathrm{C} 2 \mathrm{C} 12$ were grown for $48 \mathrm{~h}$ at low confluency in growth medium for the myoblast (MB) stage or allowed to reach $80 \%$ confluency then transferred to differentiation medium (DMEM, 2\% horse serum and $2 \%$ of insulin transferrin and selenium solution) for an additional $48 \mathrm{~h}$ for the for myotube (MT) stage. C2C12 MBs and MTs were processed for chromatin immunoprecipitation (ChIP) sequencing. For satellite cell (SC) culture, cells were maintained in a growth medium consisting of F-10 medium (Invitrogen) supplemented with 20\% heatinactivated FBS (Invitrogen), $2.5 \mathrm{ng} / \mathrm{ml} \mathrm{bFGF} \mathrm{(Millipore)} \mathrm{and} \mathrm{2 \%} \mathrm{penicillin} \mathrm{and} \mathrm{streptomycin.}$ SCs were seeded in tissue culture plates coated with type I collagen (Beckton and Dickenson) and allowed to adhere and grow for 2 to 5 days after sorting. For differentiation experiments, SCs were cultured for 48 hours in growth medium for MB stage or cultured for 48 hours in growth medium then transferred to differentiation medium [DMEM and 5\% horse serum (Invitrogen, Gibco)] for two days for MT stage. Both myoblast and myotubes were processed for RNA extraction and immunostaining. For quiescent SCs immunostaining, freshly FACS-isolated SCs were spun on collagen-coated disks mounted on glass slides and cytospun for 5 min then immediately fixed in 4\% paraformaldehyde.

\section{RNA extraction, gene expression analyses, and RNASeq}

RNA was prepared using TRizol reagent (Invitrogen) from E18.5 dissected hind limb, intercostal and para-spinal muscles as well as muscle sections (10 X $45 \mu \mathrm{M})$ generated from adult control and injured TA muscles. For FACS isolated satellite cells total RNA was extracted with TRizol, followed by loading the aqueous phase onto the RNAaqueous micro kit (Ambion). Total RNA (0.1-0.5 $\mu \mathrm{g}$ ) was reverse transcribed using cDNA synthesis kit (Applied Biosystems). Two $\mu \mathrm{ls}$ of a 1:5 or 1:10 dilution of the synthesized cDNA was used for quantitative real-time PCR, using SyberGreen PCR MasterMix (Applied Biosystems) on the Viia7 Real-Time PCR thermocycler (Applied Biosystems). Relative mRNA levels were determined by comparing threshold cycles of amplified genes with $18 \mathrm{~S}$ using the $\triangle \mathrm{CT}$ method. The oligonucleotide sequences used as primers for real-time PCR are listed in Supplemental Table 1. For all RNASeq, cDNA libraries were generated from poly A+ purified mRNA samples using the NEBNext polyA 
mRNA magnetic isolation module and Ultra II Directional RNA Library preparation kit. All libraries were then sequenced on the Illumina HiSeq 3000. Reads were aligned using the STAR v.2.5, ${ }^{39}$ the abundance of each gene was quantified using StringTie v.1.3.3. ${ }^{40}$ and the differential gene expression was performed using DESeq2 v.1.20.0. ${ }^{41}$ The Benjamini-Hochberg correction was used to adjust for multiple comparisons and a corrected p-value (q-value) of 0.05 or less was considered statistically significant. Gene ontology (GO) analyses were conducted using the online bioinformatics resource DAVID. Specifically, differentially expressed genes with a adjusted $p$ value (qvalue) $\leq 0.05$ and a-1.5 (satellite cells and hind limb) or a 2-fold change (intercostal, back and injured muscles) over control, were used to generate the GO tables and Sup Tables 3, 4 and 5, presented in this study. Top pathways were selected based on a $\mathrm{P}$ value $\leq 0.05$.

\section{CHD4 ChIP-Seq}

For ChIP-seq, C2C12 myoblast and myotubes were cross-linked in 1\% formaldehyde and processed according to published protocols ${ }^{17}$. Briefly, cells were lysed in RIPA buffer (1x PBS, $1 \%$ NP-40, 0.5\% sodium deoxycholate, $0.1 \%$ SDS) and centrifuged at $2000 \mathrm{rpm}$ for 5 minutes. The chromatin fraction was sheared by sonication $(4 \times 30 \mathrm{sec})$ in $1.5 \mathrm{ml}$ siliconized Eppendorf tubes. The resulting sheared chromatin samples were immunoprecipitated overnight with a CHD4 antibody (Ab72418) and washed in buffer I (20 mM Tris HCl pH 8.0, $150 \mathrm{mM} \mathrm{NaCl}, 2 \mathrm{mM}$ EDTA, 0.1\% SDS, 1\% Triton X-100), buffer II (20 mM Tris $\mathrm{HCl}$ pH 8.0, $500 \mathrm{mM} \mathrm{NaCl,} 2 \mathrm{mM}$ EDTA, $0.1 \%$ SDS, 1\% Triton X-100), buffer III (10 mM Tris HCl pH 8.0, 250 mM LiCl, 1\% NP-40; 1\% sodium deoxycholate, $1 \mathrm{mM}$ EDTA) and Tris-EDTA ( $\mathrm{pH} 8.0)$. All washes were performed at $4^{\circ} \mathrm{C}$ for 5 min. Finally, cross-linking was reversed in elution buffer (100 mM sodium bicarbonate (NaHCO3), $1 \% \mathrm{SDS}$ ) at $65^{\circ} \mathrm{C}$ overnight. ChIP DNA was quantified using Picogreen (Invitrogen) and ChIP DNA libraries were generated using the Ovation NEBNext Ultra II DNA library prep Kit and NEBNext multiplex oligos for Illumina index primers (New England Biolabs Inc) then sequenced on the Illumina HiSeq 3000. Data from at least two independent runs were trimmed using Trimomatic 0.38 and mapped to the mouse genome (mm10 assembly) using bowtie 2.2.4 The peaks were detected by using MACS 2.1.2 normalized to input and annotated by using Homer 4.8. Gene tracks were visualized with IGV Browser. 


\section{Acknowledgments}

This research was supported in part by the Intramural Research Program of the National Institute of Arthritis and Musculoskeletal and Skin Diseases of the National Institutes of Health.

\section{Author contributions}

A.D., K.G., and A.L.M. designed the study. A.D., W.H., C.P., K.P., and V.J.H. performed animal and laboratory experiments. A.D. and I.P.-F. performed the bioinformatic analyses. A.D. and A.L.M. drafted the manuscript. A.D., I.P.-F., W.H., C.P., K.P., V.J.H., K.G., and A.L.M. reviewed and revised the manuscript.

\section{Declaration of Interests}

The authors declare no competing interests. 


\section{Figure Legends}

Figure 1. Deletion of CHD4 in satellite cells during myogenesis. A. PCR analyses of genomic DNA from E18.5 tail muscle shows detection of the WT and CHD4 deleted KO alleles. B. Distribution of KO vs. WT/heterozygous (Het) genotypes in E18.5 mice. C. Gross morphology of CHD4 WT and KO mice at E18.5. D. CHD4 and laminin immunostaining of WT and CHD4mKO muscle at E18.5. Arrowheads indicate CHD4-negative nuclei within myofibers in the CHD4mKO. E. H\&E staining of intercostal (left panel, $a=$ lung, $b=$ diaphragm, $c=I C$ muscle) and thoracic back (right panel) E18.5 WT and CHD4mKO muscles shown at 10X (upper panel) and 40X (lower panel) magnifications. Gene ontology analyses (top panel) and gene set enrichment (lower panel) of differentially expressed genes of intercostal (F) and back muscles (G) of E18.5 CHD4mKO mice.

Figure 2. CHD4 expression in WT and CHD4 KO adult mouse satellite cells (SCs). A. Immunostaining of freshly isolated FACS-sorted WT SCs with CHD4 and Pax7. B. Immunostaining of FACS sorted WT SCs cultured in growth medium for 48 hours (myoblast stage, $\mathrm{MB}$ ) and in differentiation medium (myotube stage, MT), shows co-localization of CHD4 and Myog in MB (upper panel) and reduced CHD4 levels in nuclei of multinucleated MT stained with Myhc (lower panel). C. Schematic representation of mice crossings for the generation of the tamoxifen CRE-inducible CHD4mKO (right panel) and the intraperitoneal (IP) tamoxifen injection regiment (Day 1-4) in adult mice followed by isolation of SCs at day 15 and day 21 post IP (right panel). D. The total number of SCs sorted from WT and Tam-CRE CHD4mKO animals represented as \% of total viable cells. E RT-PCR analysis of CHD4 in WT and CHD4mKO SCs cultured in growth medium for 48 hours (myoblast stage). Stars indicate $p$ values of $p \leq 0.05$. F. Immunostaining of CHD4 in WT and Tam-CRE CHD4mKO SCs cultured in growth medium for 48 hours (myoblast stage). Arrow heads indicate CHD4-negative nuclei in CHD4 SCs from TamCRE CHD4mKO mice. G. Gene ontology analyses of differentially expressed genes in WT and Tam-CRE CHD4mKO sorted SCs cultured in growth medium for 48 hours (myoblast stage). $\mathbf{H}$. Gene ontology analyses of differentially expressed genes in Tam-CRE CHD4mKO SCs cultured in differentiation medium for 2 days (myotube stage). 
Figure 3. ChIP-Seq analysis of CHD4 binding in myoblasts and myotubes. A. Distribution of genomic regions bound by CHD4 in C2C12 myoblasts (MB) and myotubes (MT). B-D. Tracks with enriched binding of CHD4 over input DNA at (B) Wnt/ $\beta$-catenin (C), lipid metabolism (D), and hemoglobin (E) genes.

Figure 4. Abnormal muscle regeneration in adult Tam-CRE CHD4 conditional KO mice. A. Schematic representation showing intraperitoneal (IP) tamoxifen injections for four consecutive days (D1-D4) followed by cardiotoxin (CTX) injury and collection of the injured TA muscle at days 7 through 28 (CTX D7, CTX D10, CTX D14, and CTX D28) post CTX. B. CHD4 and laminin immunostaining of WT and CHD4 KO cardiotoxin injured TA (CTX D10) muscle cross sections. Arrowheads indicate CHD4-negative nuclei within CHD4 regenerating myofibers in the Tam-CRE CHD4mKO TA muscle. C. Quantitative analysis shows the percentage of CHD4positive regenerating myofibers in the Tam-CRE CHD4mKO mice relative to WT controls. D.

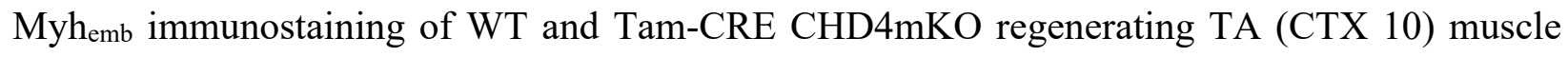
cross sections. E. Laminin-stained myofibers of WT and Tam-CRE CHD4mKO injured TA muscles (CTX 10). F. Fiber diameter size distribution in regenerating muscle fibers from TamCRE CHD4 KO (red) and WT (blue) mice at CTX D14 and CTX D28. G. Bar graph representation of fiber diameter size distribution in WT and Tam-CRE CHD4mKO injured TA muscles at CTX D28. H. Bar graph representation of total myofiber number in WT and Tam-CRE CHD4mKO injured TA muscles at CTX D28. Stars indicate $\mathrm{p}$ values of $\mathrm{p} \leq 0.05$.

Supplemental Figure 1. Deletion of CHD4 in satellite cells during myogenesis. H\&E staining of E18.5 WT and CHD4mKO hindlimb (A) and (B) cranial thorax muscles shown at 10X (upper panel) and 40X (lower panel) magnifications. Stars indicate compared muscles. C. Venn diagram of differentially ( $P$ adj value $\leq 0.05)$ expressed genes in the intercostal, back, and hindlimb muscles of CHD4mKO mice. D. Gene ontology analyses of differentially expressed genes in the hindlimb muscles of E18.5 CHD4mKO mice. 


\section{References}

1 O'Shaughnessy, A. \& Hendrich, B. CHD4 in the DNA-damage response and cell cycle progression: not so NuRDy now. Biochem Soc Trans 41, 777-782, (2013).

2 Williams, C. J. et al. The chromatin remodeler Mi-2beta is required for CD4 expression and T cell development. Immunity 20, 719-733, (2004).

3 Saether, T. et al. The chromatin remodeling factor Mi-2alpha acts as a novel co-activator for human c-Myb. J Biol Chem 282, 13994-14005, (2007).

4 Miccio, A. et al. NuRD mediates activating and repressive functions of GATA-1 and FOG1 during blood development. EMBO J 29, 442-456, (2010).

5 Hoffmeister, H. et al. CHD3 and CHD4 form distinct NuRD complexes with different yet overlapping functionality. Nucleic Acids Res 45, 10534-10554, (2017).

6 O'Shaughnessy-Kirwan, A., Signolet, J., Costello, I., Gharbi, S. \& Hendrich, B. Constraint of gene expression by the chromatin remodelling protein CHD4 facilitates lineage specification. Development 142, 2586-2597, (2015).

7 Kashiwagi, M., Morgan, B. A. \& Georgopoulos, K. The chromatin remodeler Mi-2beta is required for establishment of the basal epidermis and normal differentiation of its progeny. Development 134, 1571-1582, (2007).

8 Yamada, T. et al. Promoter decommissioning by the NuRD chromatin remodeling complex triggers synaptic connectivity in the mammalian brain. Neuron 83, 122-134, (2014).

9 Yang, Y. et al. Chromatin remodeling inactivates activity genes and regulates neural coding. Science 353, 300-305, (2016).

10 Yoshida, T. et al. Chromatin restriction by the nucleosome remodeler Mi-2beta and functional interplay with lineage-specific transcription regulators control B-cell differentiation. Genes Dev 33, 763-781, (2019).

11 Mammen, A. L. et al. Expression of the dermatomyositis autoantigen Mi-2 in regenerating muscle. Arthritis Rheum 60, (2009).

12 Pinal-Fernandez, I. et al. Myositis Autoantigen Expression Correlates With Muscle Regeneration but Not Autoantibody Specificity. Arthritis Rheumatol 71, 1371-1376, (2019).

13 Sreenivasan, K. et al. Attenuated Epigenetic Suppression of Muscle Stem Cell Necroptosis Is Required for Efficient Regeneration of Dystrophic Muscles. Cell Rep 31, (2020). 
14 Gomez-Del Arco, P. et al. The Chromatin Remodeling Complex Chd4/NuRD Controls Striated Muscle Identity and Metabolic Homeostasis. Cell Metab 23, 881-892, (2016).

15 Keller, C., Hansen, M. S., Coffin, C. M. \& Capecchi, M. R. Pax3:Fkhr interferes with embryonic Pax3 and Pax7 function: implications for alveolar rhabdomyosarcoma cell of origin. Genes Dev 18, 2608-2613, (2004).

16 von Maltzahn, J., Renaud, J. M., Parise, G. \& Rudnicki, M. A. Wnt7a treatment ameliorates muscular dystrophy. Proc Natl Acad Sci U S A 109, 20614-20619, (2012).

17 Juan, A. H. et al. Polycomb EZH2 controls self-renewal and safeguards the transcriptional identity of skeletal muscle stem cells. Genes Dev 25, 789-794, (2011).

18 Brack, A. S., Conboy, I. M., Conboy, M. J., Shen, J. \& Rando, T. A. A temporal switch from notch to Wnt signaling in muscle stem cells is necessary for normal adult myogenesis. Cell Stem Cell 2, 50-59, (2008).

19 Murphy, M. M. et al. Transiently active Wnt/beta-catenin signaling is not required but must be silenced for stem cell function during muscle regeneration. Stem Cell Reports 3, 475488, (2014).

20 Costa, F. C., Fedosyuk, H., Chazelle, A. M., Neades, R. Y. \& Peterson, K. R. Mi2beta is required for gamma-globin gene silencing: temporal assembly of a GATA-1-FOG-1-Mi2 repressor complex in beta-YAC transgenic mice. PLoS Genet 8, e1003155, (2012).

21 Harju-Baker, S., Costa, F. C., Fedosyuk, H., Neades, R. \& Peterson, K. R. Silencing of Agamma-globin gene expression during adult definitive erythropoiesis mediated by GATA-1-FOG-1-Mi2 complex binding at the -566 GATA site. Mol Cell Biol 28, 31013113, (2008).

22 Sankaran, V. G. et al. Human fetal hemoglobin expression is regulated by the developmental stage-specific repressor BCL11A. Science 322, 1839-1842, (2008).

$23 \mathrm{Xu}, \mathrm{J}$. et al. Transcriptional silencing of \{gamma $\}$-globin by BCL11A involves long-range interactions and cooperation with SOX6. Genes Dev 24, 783-798, (2010).

24 Kablar, B. \& Rudnicki, M. A. Skeletal muscle development in the mouse embryo. Histol Histopathol 15, 649-656, (2000).

25 Gamer, L. W., Cox, K. A., Small, C. \& Rosen, V. Gdf11 is a negative regulator of chondrogenesis and myogenesis in the developing chick limb. Dev Biol 229, 407-420, (2001). 
26 Egerman, M. A. et al. GDF11 Increases with Age and Inhibits Skeletal Muscle Regeneration. Cell Metab 22, 164-174, (2015).

27 Hammers, D. W. et al. Supraphysiological levels of GDF11 induce striated muscle atrophy. EMBO Mol Med 9, 531-544, (2017).

28 Bhanot, P. et al. A new member of the frizzled family from Drosophila functions as a Wingless receptor. Nature 382, 225-230, (1996).

29 Cadigan, K. M. \& Nusse, R. Wnt signaling: a common theme in animal development. Genes Dev 11, 3286-3305 (1997).

30 Clevers, H. Wnt/beta-catenin signaling in development and disease. Cell 127, 469-480, (2006).

31 von Maltzahn, J., Chang, N. C., Bentzinger, C. F. \& Rudnicki, M. A. Wnt signaling in myogenesis. Trends Cell Biol 22, 602-609, (2012).

32 Wilczewski, C. M. et al. CHD4 and the NuRD complex directly control cardiac sarcomere formation. Proc Natl Acad Sci US A 115, 6727-6732, (2018).

33 Lassar, A. B., Skapek, S. X. \& Novitch, B. Regulatory mechanisms that coordinate skeletal muscle differentiation and cell cycle withdrawal. Curr Opin Cell Biol 6, 788-794, (1994).

34 Helbling Chadwick, L., Chadwick, B. P., Jaye, D. L. \& Wade, P. A. The Mi-2/NuRD complex associates with pericentromeric heterochromatin during $\mathrm{S}$ phase in rapidly proliferating lymphoid cells. Chromosoma 118, 445-457, (2009).

35 Sims, J. K. \& Wade, P. A. Mi-2/NuRD complex function is required for normal S phase progression and assembly of pericentric heterochromatin. Mol Biol Cell 22, 3094-3102, (2011).

36 Carlson, M. E., Hsu, M. \& Conboy, I. M. Imbalance between pSmad3 and Notch induces CDK inhibitors in old muscle stem cells. Nature 454, 528-532, (2008).

37 Jones, A. E. et al. Wnt/beta-catenin controls follistatin signalling to regulate satellite cell myogenic potential. Skelet Muscle 5, 14, (2015).

38 Aussy, A., Boyer, O. \& Cordel, N. Dermatomyositis and Immune-Mediated Necrotizing Myopathies: A Window on Autoimmunity and Cancer. Front Immunol 8, 992, 2 (2017).

39 Dobin, A. et al. STAR: ultrafast universal RNA-seq aligner. Bioinformatics 29, 15-21, (2013). 
40 Pertea, M. et al. StringTie enables improved reconstruction of a transcriptome from RNAseq reads. Nat Biotechnol 33, 290-295, (2015).

41 Love, M. I., Huber, W. \& Anders, S. Moderated estimation of fold change and dispersion for RNA-seq data with DESeq2. Genome Biol 15, 550, (2014). 
Figure 1. Deletion of CHD4 in satellite cells during myogenesis.

A.

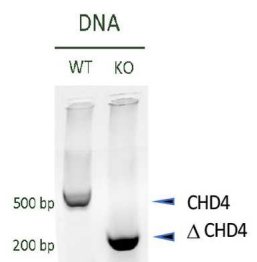

D

E.

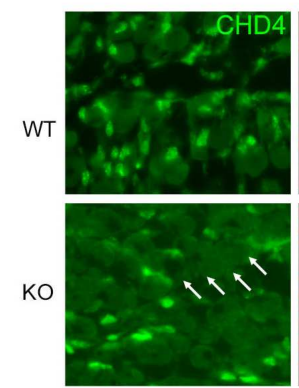

WT

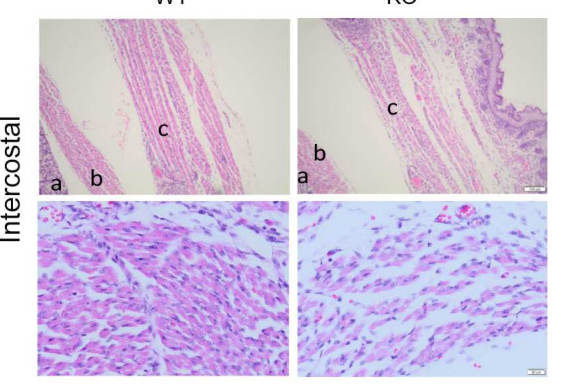

F.

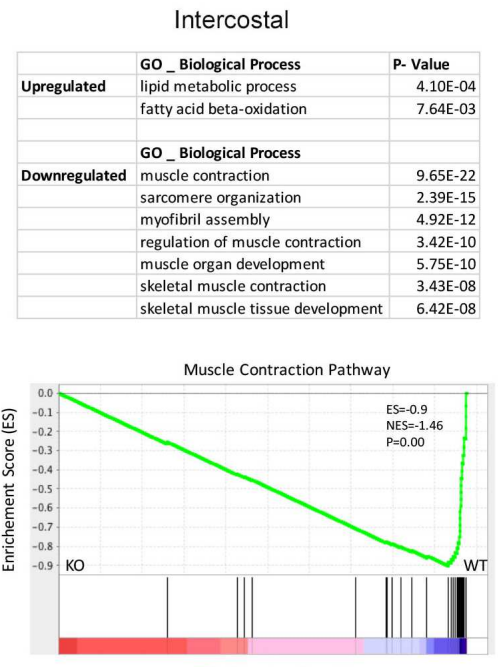

B.

KO

\begin{tabular}{|l|c|c|}
\hline Genotype & Number & $\%$ \\
\hline KO & 26 & 25.2 \\
\hline WT/Het & 77 & 74.8 \\
\hline Total & 103 & 100 \\
\hline
\end{tabular}

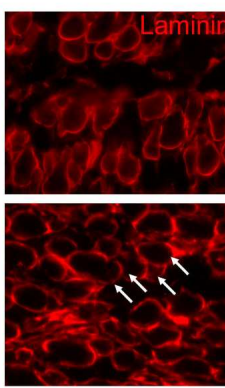

G.
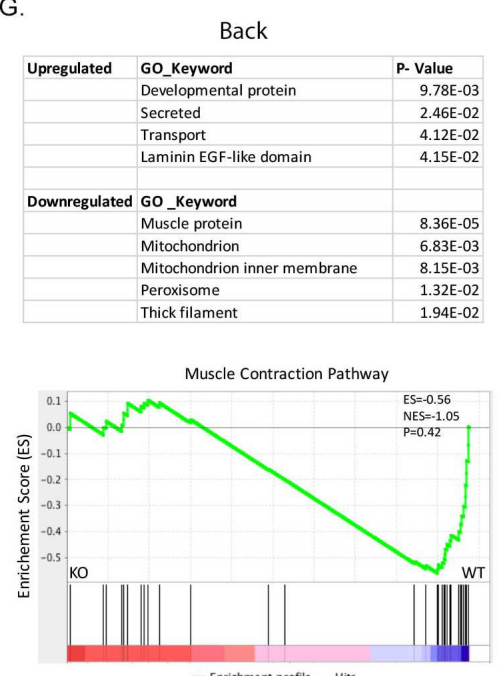

C
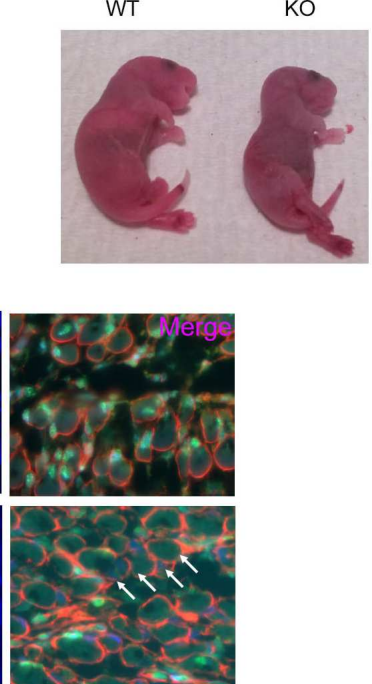

KO

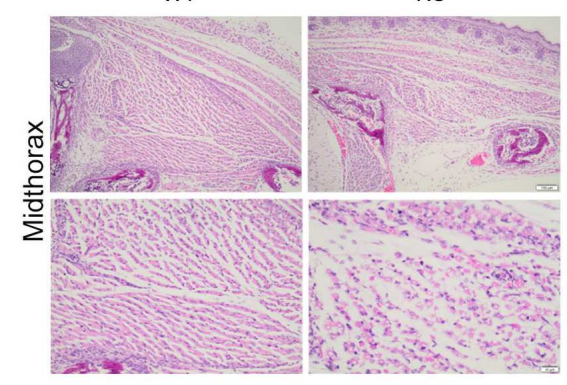

Enrichment profile - Hits 
Figure 2. CHD4 expression in WT and CHD4 KO adult mouse satellite cells.

A.
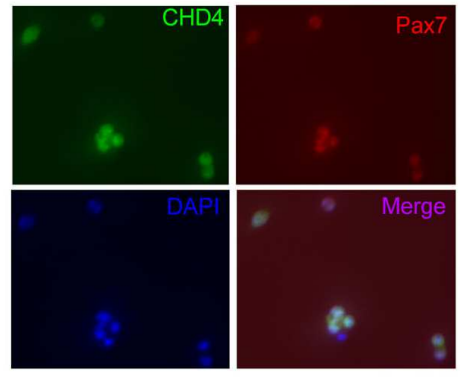

c.

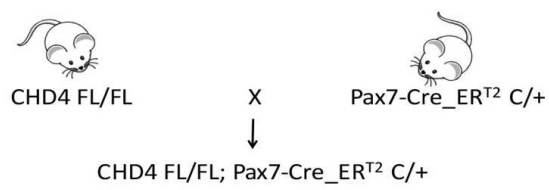

$D$

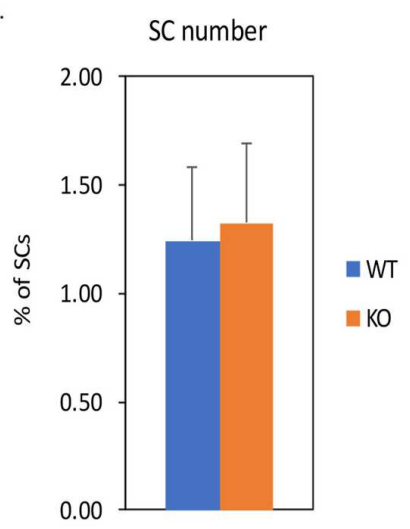

B.

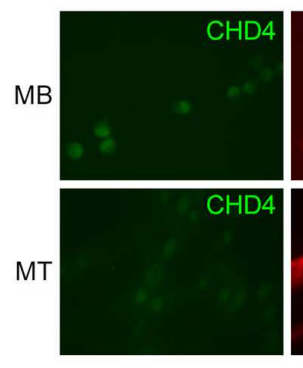

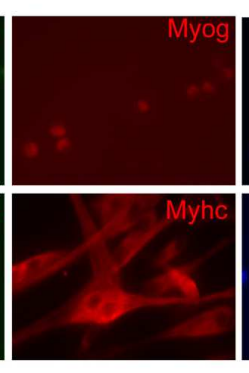
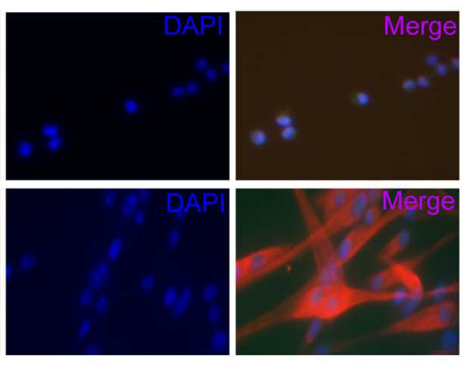

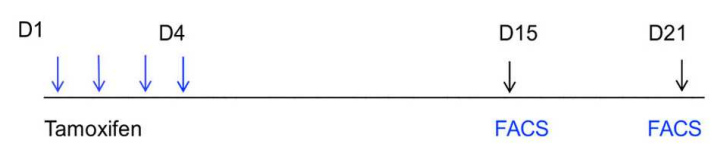

F.

CHD4 RNA in SCs
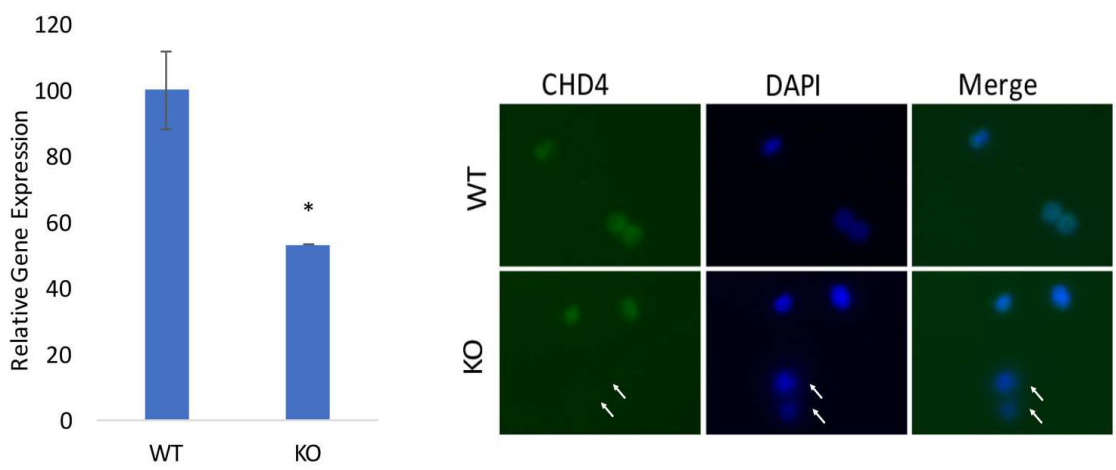

H.

Myotubes

\begin{tabular}{|l|l|r|}
\hline Upregulated & GO_Cellular Component & P-Value \\
\hline & cell surface & $2.99 \mathrm{E}-13$ \\
\hline & extracellular matrix & $3.10 \mathrm{E}-12$ \\
\hline & proteinaceous extracellular matrix & $9.14 \mathrm{E}-11$ \\
\hline & extracellular exosome & $3.46 \mathrm{E}-09$ \\
\hline & extracellular region & $7.91 \mathrm{E}-09$ \\
\hline & & \\
\hline Downregulated & GO_Cellular Component & P-Value \\
\hline & mitochondrion & $1.28 \mathrm{E}-09$ \\
\hline mitochondrial inner membrane & $4.77 \mathrm{E}-07$ \\
\hline myofibril & $1.55 \mathrm{E}-05$ \\
\hline Z disc & $5.99 \mathrm{E}-05$ \\
\hline mitochondrial matrix & $1.31 \mathrm{E}-04$ \\
\hline
\end{tabular}


Figure 3. ChIP-Seq analysis of CHD4 binding in myoblasts and myotubes.

A.

\begin{tabular}{|l|r|r|}
\hline & MB & MT \\
\hline Promoter & $2.6 \%$ & $15.2 \%$ \\
TSS & $2.9 \%$ & $2.2 \%$ \\
Exon & $2.3 \%$ & $1.6 \%$ \\
3'UTR & $1.0 \%$ & $0.8 \%$ \\
Intron & $16.2 \%$ & $26.7 \%$ \\
Intergenic & $74.4 \%$ & $52.6 \%$ \\
Non coding & $0.6 \%$ & $1.0 \%$ \\
\hline
\end{tabular}

C.

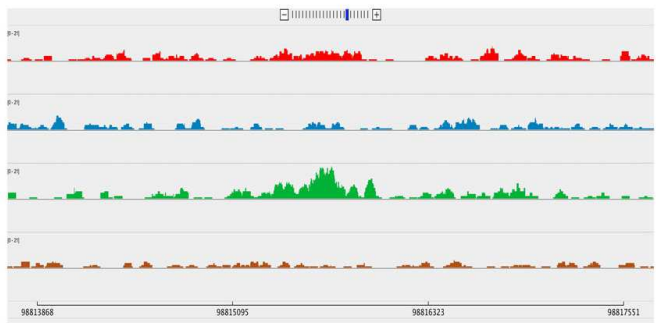

D.

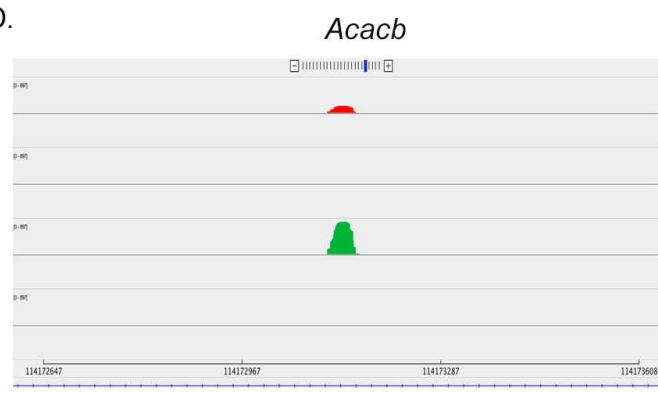

E.

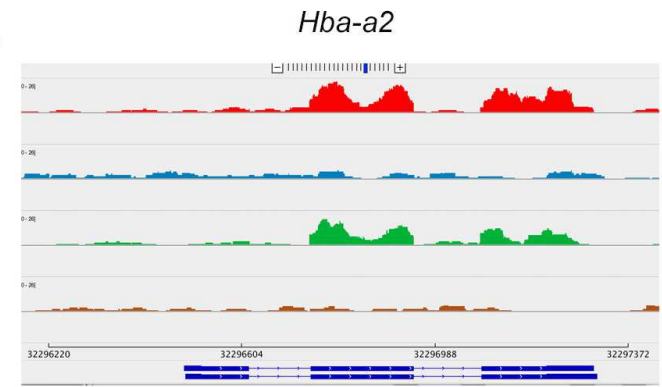

B.
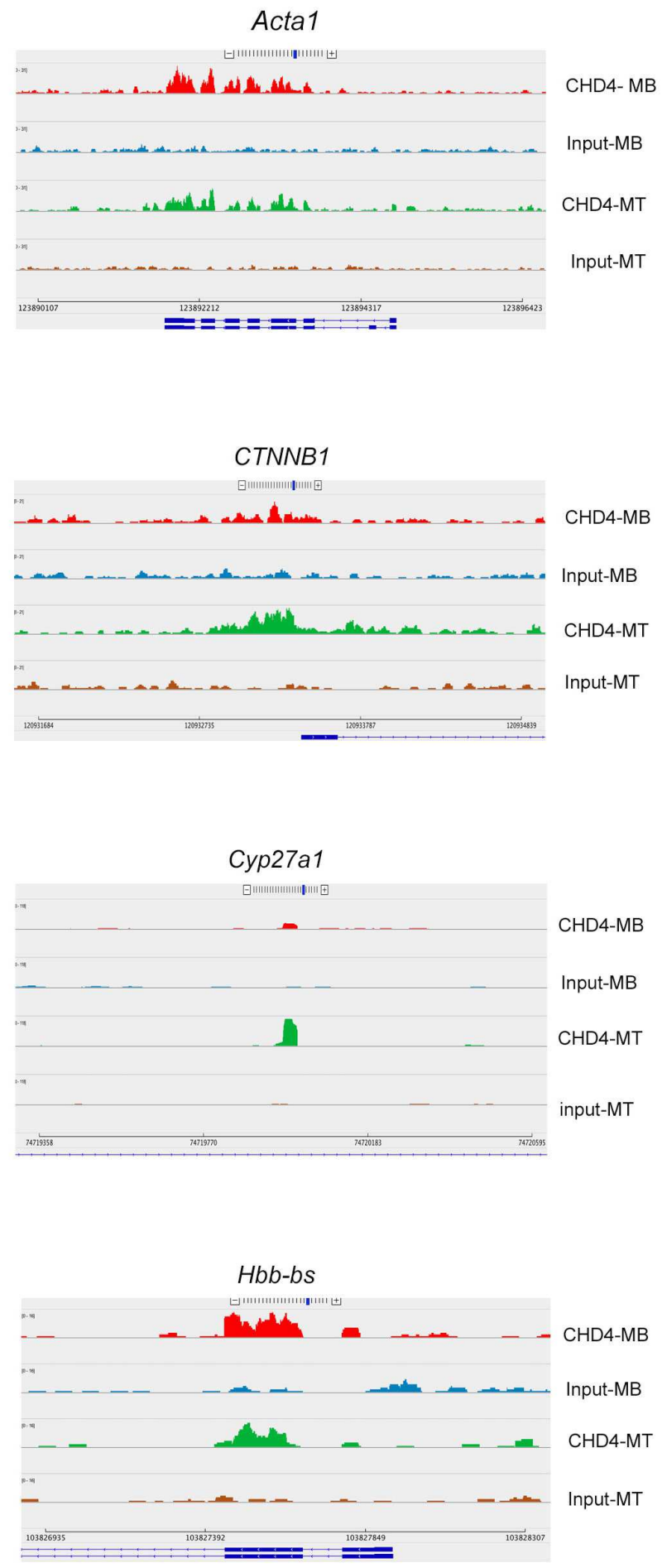
Figure 4. Abnormal muscle regeneration in adult Tam-CRE CHD4 conditional KO mice.

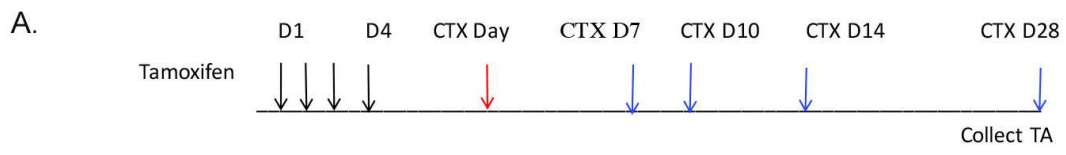

B.

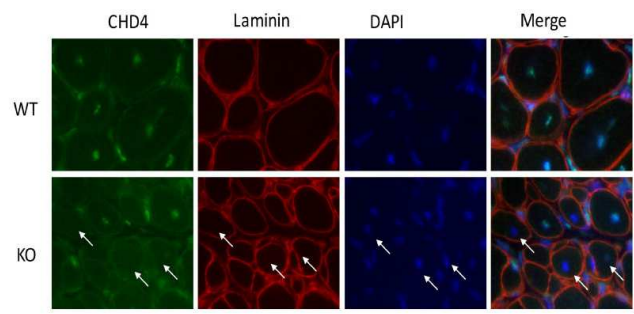

E.

$$
\text { CTX D10 }
$$

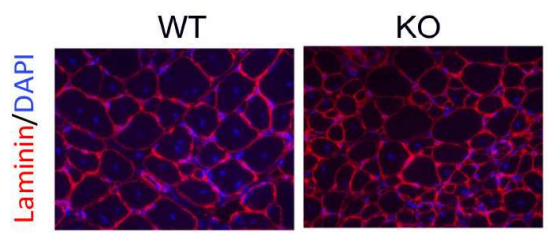

G.

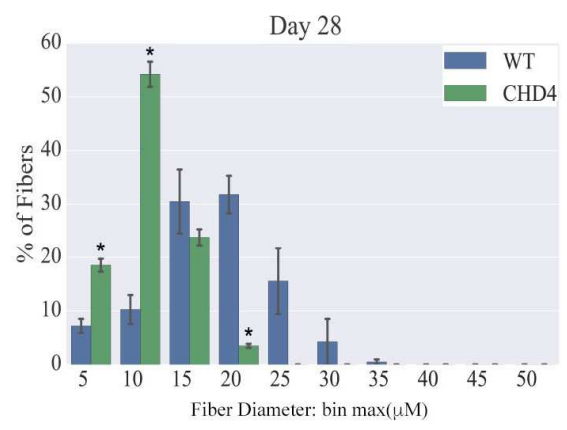

C.

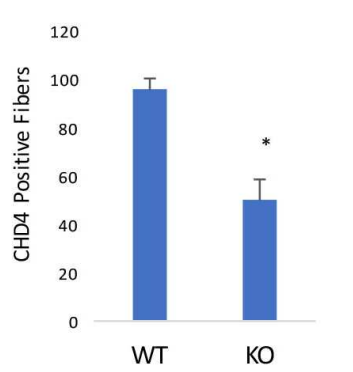

F.
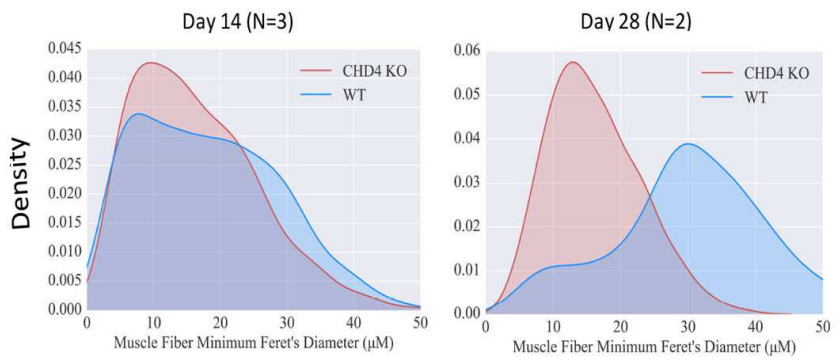

$\mathrm{H}$.

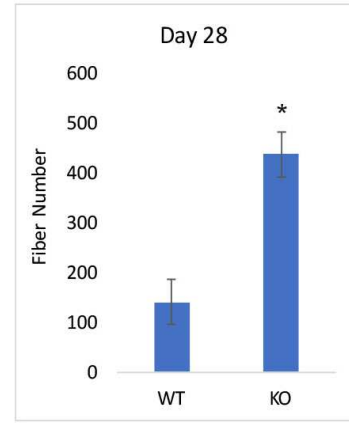


Figures

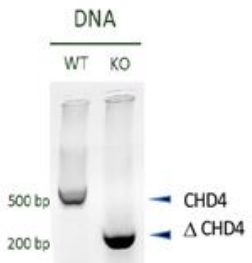

\begin{tabular}{|l|c|c|}
\hline Genotype & Number & $\%$ \\
\hline KO & 26 & 25.2 \\
\hline WT/Het & 77 & 74.8 \\
\hline Total & 103 & 100 \\
\hline
\end{tabular}

C.

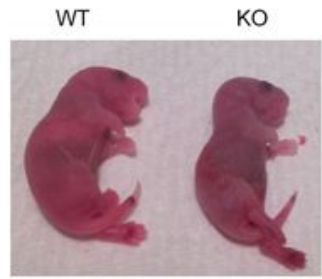

D
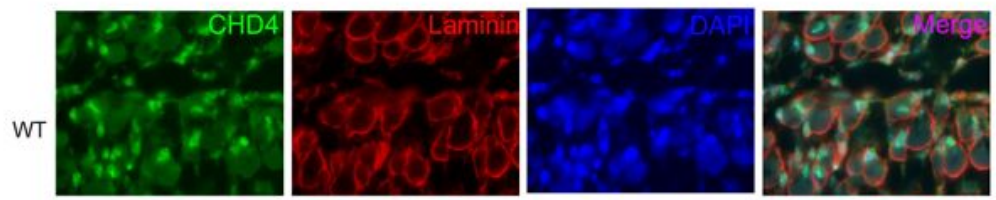

KO
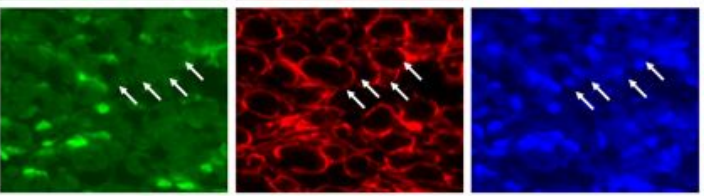

KO
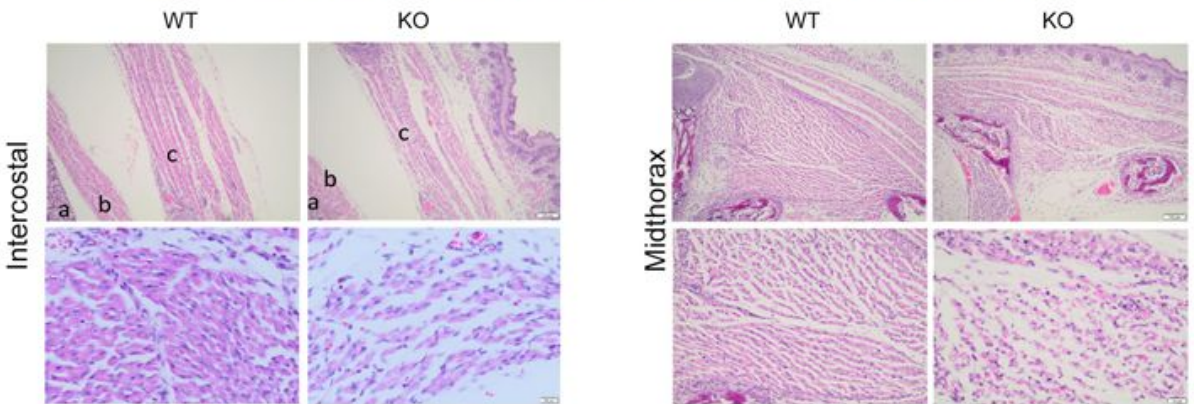

G.

F.

Intercostal
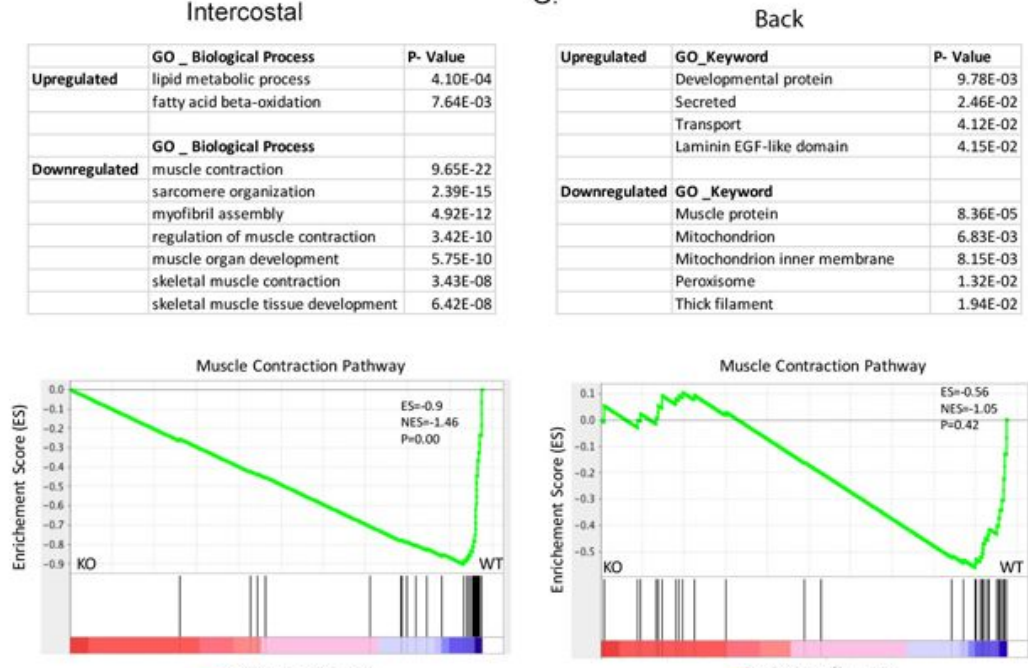

Figure 1

Deletion of CHD4 in satellite cells during myogenesis. 
A.

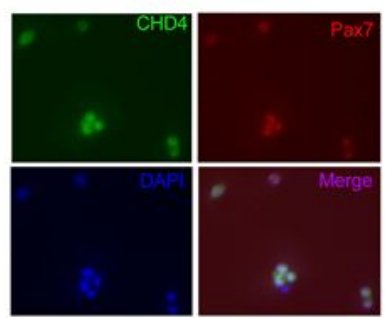

B.

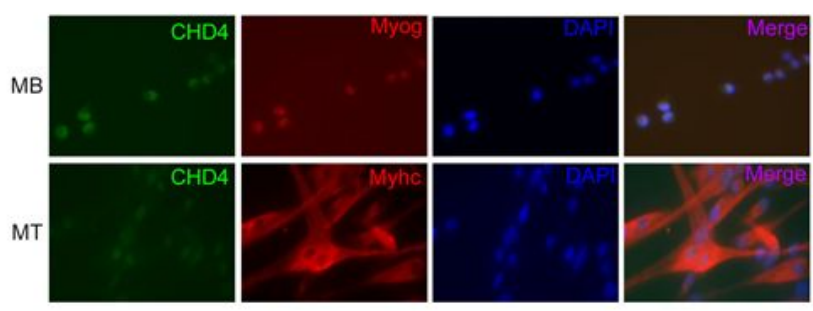

C.
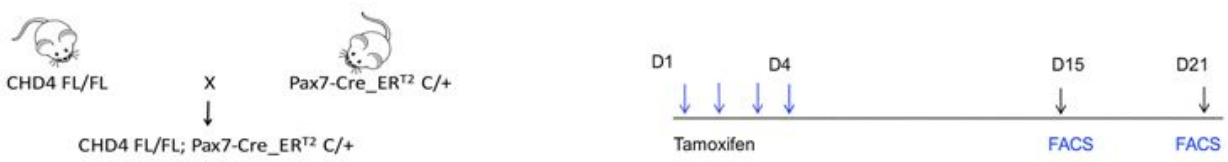

D.

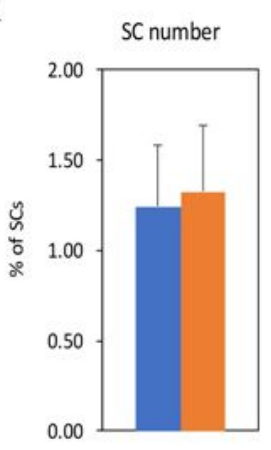

E.

F.

G

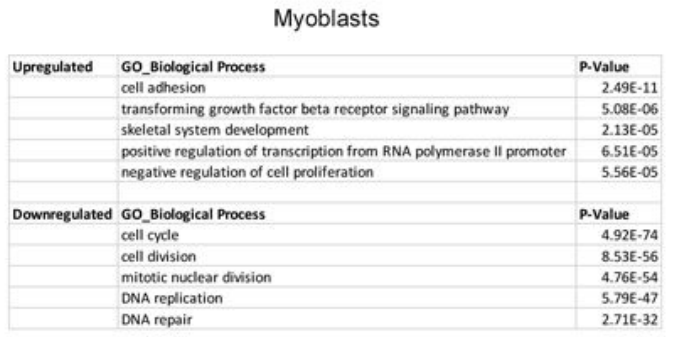

CHD4 RNA in SCs
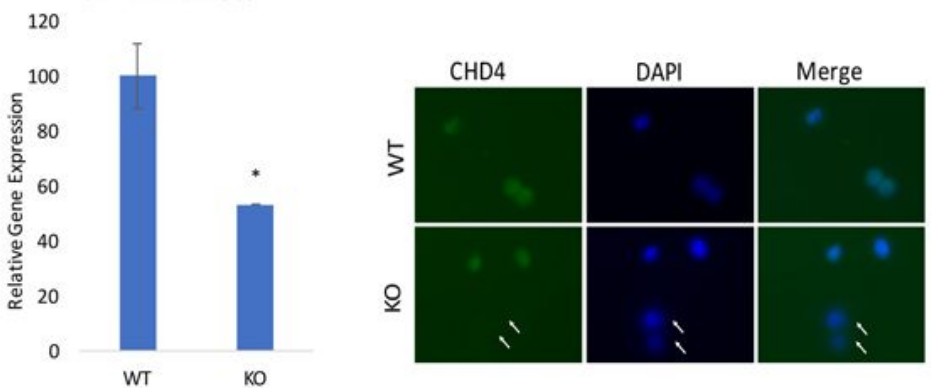

$\mathrm{H}$.

Myotubes

\begin{tabular}{|c|c|c|}
\hline \multirow{6}{*}{ Upregulated } & Go_Cellular Component & P.Value \\
\hline & cell surface & $2.99 \mathrm{E}-13$ \\
\hline & extracellular matrix & $3.10 \mathrm{E}-12$ \\
\hline & proteinaceous extracellular matrix & $9.14 \mathrm{E}-11$ \\
\hline & extracellular exosome & $3.46 \mathrm{E}-09$ \\
\hline & extracellular region & $7.91 \mathrm{E}-09$ \\
\hline \multirow{6}{*}{ Downregulated } & Go_Cellular Component & P.Value \\
\hline & mitochondrion & 1.28E-09 \\
\hline & mitochondrial inner membrane & $4.77 \mathrm{E}-07$ \\
\hline & myofibril & $1.55 \mathrm{E}-05$ \\
\hline & 2 disc & $5.99 \mathrm{E}-05$ \\
\hline & mitochondrial matrix & $1.31 \mathrm{E}-04$ \\
\hline
\end{tabular}

Figure 2

CHD4 expression in WT and CHD4 KO adult mouse satellite cells. 
A.

\begin{tabular}{|l|r|r|}
\hline & MB & MT \\
\hline Promoter & $2.6 \%$ & $15.2 \%$ \\
TSS & $2.9 \%$ & $2.2 \%$ \\
Exon & $2.3 \%$ & $1.6 \%$ \\
3'UTR & $1.0 \%$ & $0.8 \%$ \\
Intron & $16.2 \%$ & $26.7 \%$ \\
Intergenic & $74.4 \%$ & $52.6 \%$ \\
Non coding & $0.6 \%$ & $1.0 \%$ \\
\hline
\end{tabular}

c.

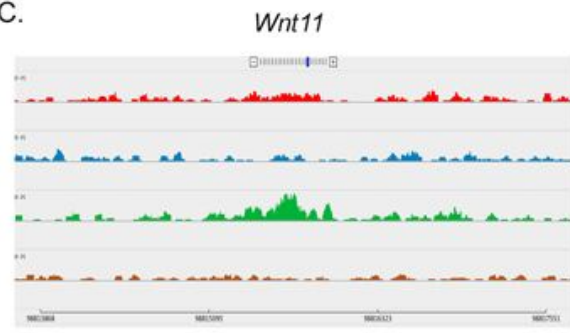

D.

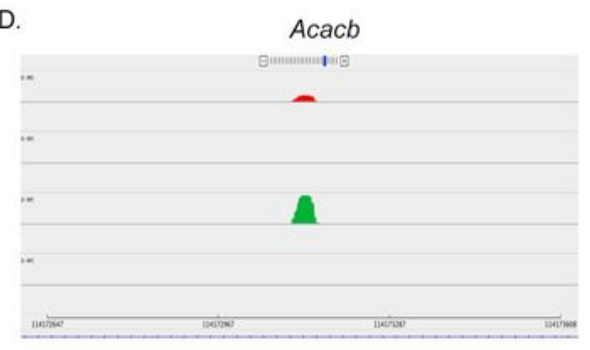

E.

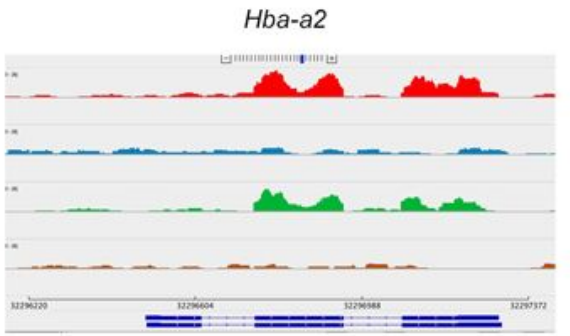

B.
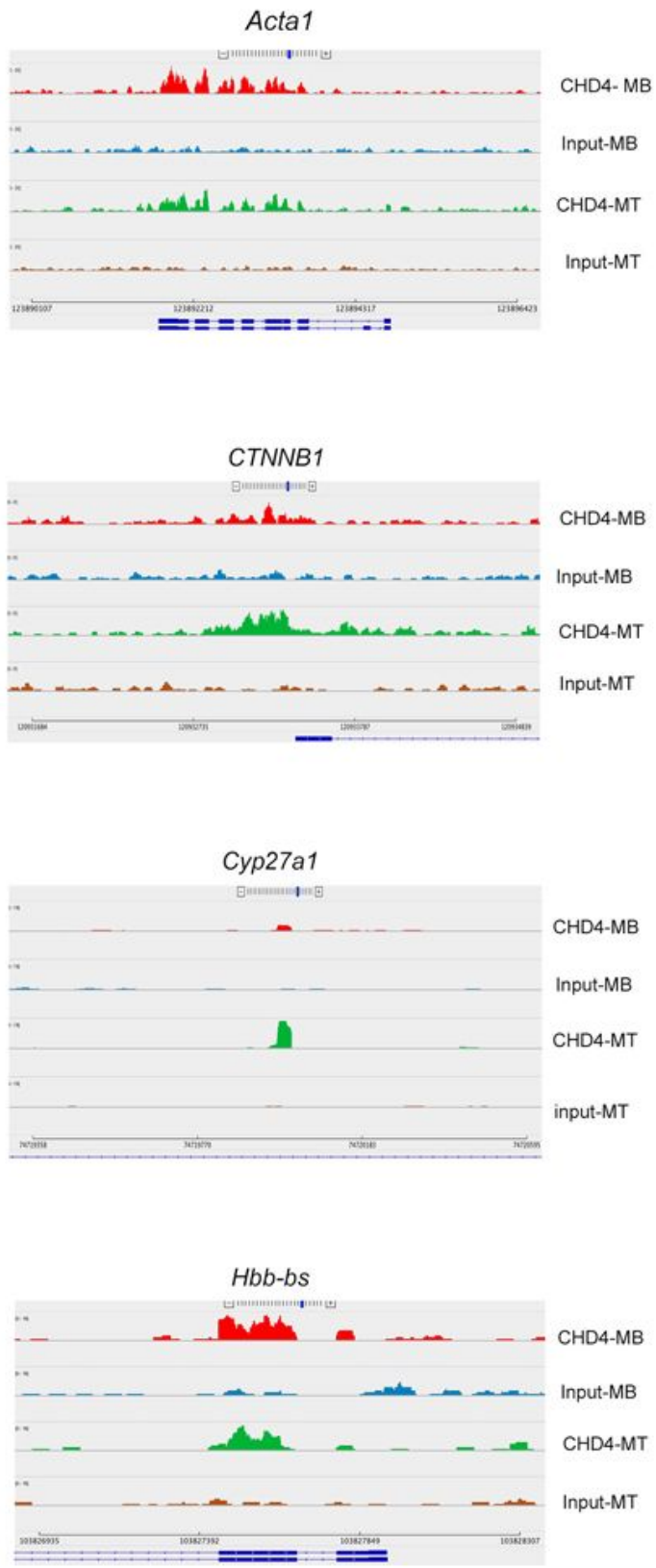

\section{Figure 3}

ChIP-Seq analysis of CHD4 binding in myoblasts and myotubes. 


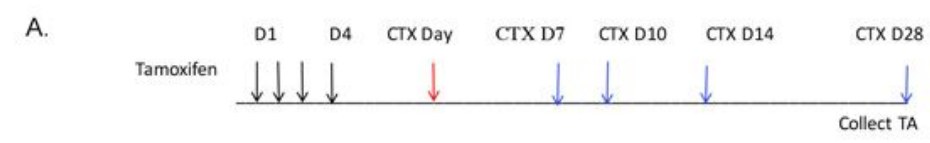

B.

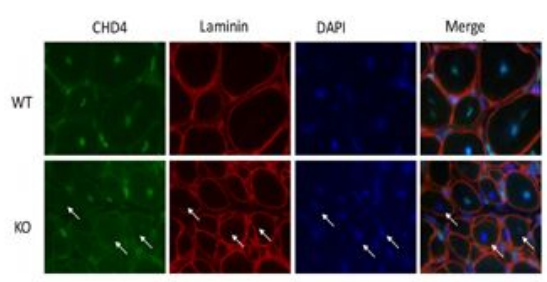

E.

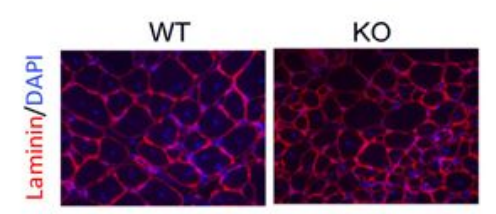

G.

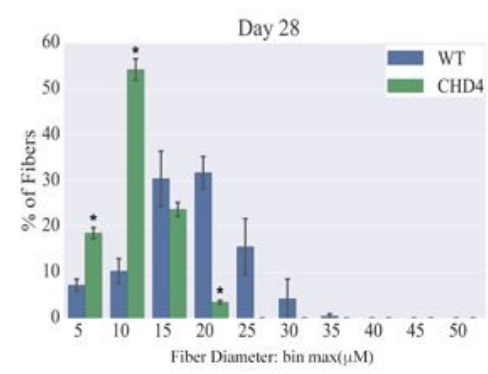

C.

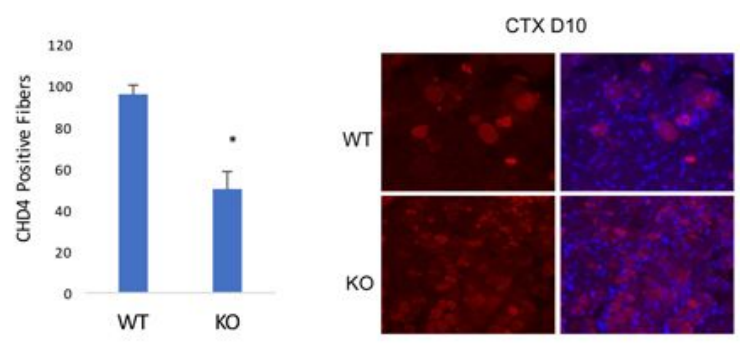

F.
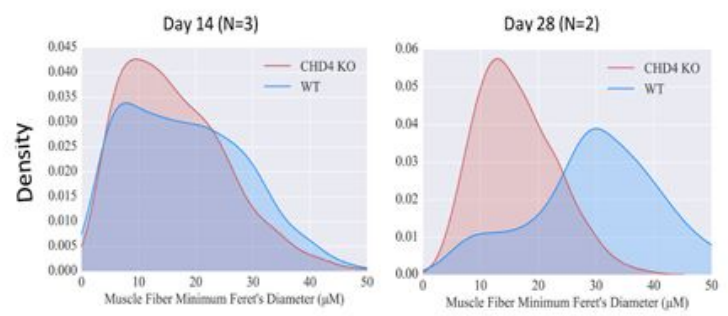

H.

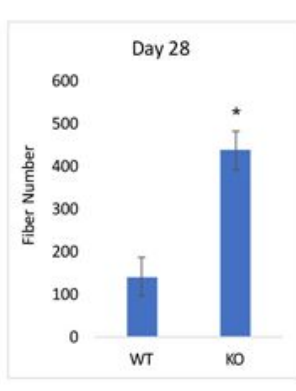

\section{Figure 4}

Abnormal muscle regeneration in adult Tam-CRE CHD4 conditional KO mice.

\section{Supplementary Files}

This is a list of supplementary files associated with this preprint. Click to download. 
- Supplemmentarylnfo20201124.pdf 